\title{
Disease Genes and Gene Regulation by microRNAs
}

\author{
Robert Roberts $\cdot$ Clifford J. Steer
}

Received: 31 March 2010 / Accepted: 5 April 2010 /Published online: 22 April 2010

(C) Springer Science+Business Media, LLC 2010

The approach to biology and genetics has been markedly influenced by recent discoveries, namely, non-proteincoding RNAs [1], the annotation of single nucleotide polymorphisms (SNPs) by the HapMap Project [2], and the assembly of these SNPs onto computerized chips (microarrays) to pursue genome-wide association studies [3], This issue discusses the application and progress of these new discoveries as they relate to the biology and genetics of disease.

The application of genetics to the pursuit of genes responsible for single-gene disorders received a major boost in the 1980s. The work of Nakamura et al. [4] and Murray et al. [5] made available hundreds of highly informative DNA markers consisting of short repeats that span the human genome. These markers coupled with polymerase chain reaction greatly accelerated mapping the chromosomal location (locus) of genes responsible for disease. The past two decades have been the golden age for single-gene disorders. It is estimated there are 6,000 inherited singlegene disorders, of which the genes for more than 2,000 have been identified [6]. However, single-gene disorders are rare and have a prevalence of less than one tenth of $1 \%$. In contrast, diseases such as coronary artery disease (CAD), the number one killer, are common yet have a large genetic

\footnotetext{
R. Roberts

The John \& Jennifer Ruddy

Canadian Cardiovascular Genetics Centre,

Ottawa, ON, Canada K1Y 4W7

e-mail: rroberts@ottawaheart.ca

C. J. Steer $(\bowtie)$

Department of Medicine,

University of Minnesota Medical School,

Minneapolis, MN 55455, USA

e-mail: steer001@umn.edu
}

component. The technology to pursue the mapping of loci associated with common diseases would have to wait. These common diseases are polygenic with multiple genes contributing to their genetic predisposition; thus, each gene exerts only minimal effect on the phenotype. Thus, several genes acting in concert are required to induce the phenotype. To map the chromosomal locus of a gene with minimal effect on the phenotype requires not hundreds of DNA markers but hundreds of thousands of DNA markers. It also requires thousands of unrelated cases and controls analyzed for gene frequency in cases versus controls, referred to as a case-control association study. The preferred approach is to genotype with hundreds of thousands of markers selected to span the whole genome. Then, in an unbiased fashion, one can determine which markers are more common in cases (risk variant) or more common in controls (protective variant). In 2005, the technology arrived in the form of a microarray with 500,000 SNPs [7] and the necessary high throughput platform [3]. In 2007, two groups simultaneously mapped the first risk variant, 9p21, for CAD [8] and myocardial infarction [9]. The pursuit was intense, and in just 5 years, more than 400 chromosomal loci have been mapped to be associated with disease [10].

The case-control association study method and its genome-wide application referred to as genome-wide association study (GWAS) has been a remarkable success. The GWAS method has been dissected and analyzed in three reviews of this issue. The authors analyzed the advantages and disadvantages of the GWAS and provide a progress report on their application to cardiovascular disease in particular, CAD and hypertension. The ultimate application of these loci in the prevention, diagnoses and treatment of disease will require identification of the causative sequence and its function. We have already been 
surprised, namely, most loci were not as expected in protein-coding DNA sequences but rather in noncoding regulatory sequences and regions of non-protein-coding RNAs. The 9p21 locus which exhibits the most robust association for $\mathrm{CAD}$ encodes a non-coding RNA referred to as ANRIL [11].

The primary role of RNA in the cell has traditionally been considered in the context of protein expression, limiting RNA to its function as mRNA, tRNA, and rRNA. The discovery of a diverse array of noncoding RNA (ncRNA) genes that functions as RNAs to regulate DNA replication, transcription, RNA processing, translation and stability of mRNAs, and even protein stability and translocation has changed this view profoundly $[12,13]$.

MicroRNAs (miRNAs) are a recently discovered class of small ncRNAs that modulate gene expression. Mature miRNAs are produced from sequential processing of primary transcripts via specific RNAses. These 18- to 24-nt-long miRNAs down-regulate protein expression of specific mRNA by either translational inhibition or mRNA degradation [14]. Numerous reports have now shown that miRNAs are differentially expressed in many types of human cancers $[15,16]$. In addition, altered miRNA expression has also been found in most disease states unrelated to cancer [17]. The molecular signaling pathways modulated by miRNAs have become a major focus of study in both normal and abnormal cell function.

Mature miRNAs are evolutionarily conserved small ncRNAs, which in general are transcribed by RNA polymerase II as primary transcripts (pri-miRNAs). More than $70 \%$ of miRNAs are located in either introns or exons of protein-coding genes and the remainder found in intergenic regions. A significant number of pri-miRNAs are expressed as polycistronic (transcript containing multiple miRNAs) RNAs [18]. The pri-miRNAs are subsequently processed by Drosha, an RNAse III enzyme to a $\sim 70$ nucleotide-long stem-loop structure called precursor miRNA (pre-miRNA). The pre-miRNAs are exported to the cytoplasm by exportin-5 and then processed as mature double-stranded miRNAs by Dicer, another RNAse III enzyme; and then bound to miRNA induced silencing complex (RISC) to form mature miRNAs 18-23 nucleotides in length. The key proteins in the complex are Argonaute 2 and transactivation-responsive RNA-binding protein. In the RISC complex, the active strand is retained and the passenger strand is selectively degraded. The mature miRNA along with RISC binds to complementary sites in the mRNA transcripts and (typically) downregulates gene expression $[14,19]$. miRNAs that bind to mRNA targets with imperfect complementarity regulate the target gene at the level of protein translation, while miRNAs that bind to their mRNA targets with perfect complementarity induce degradation $[20,21]$.
The discovery of miRNAs has dramatically changed our view of gene expression and its regulation, adding a new layer of complexity to an already intricate process. miRNAs were first discovered in 1993 in the nematode Caenorhabditis elegans. The lin-4 gene, which was known to regulate the timing of larval development in C. elegans, was found to express two small RNA transcripts, 22 and 61 nucleotides long. These small RNA molecules were shown to have antisense complementarity to the $3^{\prime} \mathrm{UTR}$ of the lin-14. In addition, it was shown that the negative regulation of lin-14 gene was due to lin-4-mediated posttranscriptional regulation, which resulted in decreased lin-14 protein, without noticeable changes in mRNA levels $[22,23]$. This pivotal discovery was not fully appreciated until 7 years later, when the 21-nucleotide let-7 RNA was discovered, again displaying complementarity to the $3^{\prime}$ UTR of several genes [24]. Importantly, let-7 is critical for C. elegans developmental timing regulation and is conserved in animals [25].

Since these initial findings, a monumental research effort has been underway to unravel the basis of miRNA biogenesis, mechanisms of action, and regulatory impact on normal and pathogenic cellular processes. It is now clear that miRNAs are abundant, evolutionary conserved, and endogenously encoded small ncRNA molecules. This has lead to the identification of more than 700 human miRNAs and many more in other mammals, worms, fish, and plants [26]. Nevertheless, this growing miRNA class is predicted by bioinformatics to include more than 1000 members [27], which may turn out to be significantly underestimated [28]. In addition, miRNAs were predicted to posttranscriptionally regulate more than $30 \%$ of human genes, which again may be a real underestimate. In fact, it was recently suggested that miRNAs might regulate more than $60 \%$ of human genes [29].

There is growing evidence to support the role of miRNAs in the regulation of many crucial biological processes including human cancer [15]. Nevertheless, the biological function of the vast majority of miRNAs remains unknown, despite the rapid rate of information that is being generated for individual miRNAs and their targets. Systemic analysis of the spatial-temporal expression of miRNAs has shown that many of these small ncRNAs have strong tissue specificity together with tight temporal expression regulation starting from early phases of embryogenesis. This fine modulation has been shown to play an important role in cell lineage commitment and embryonic tissue development by temporal activation/inactivation of specific mRNA targets.

In the cardiovascular field, miRNAs are now acknowledged as fundamental in regulating the expression of genes that govern physiological and pathological myocardial adaptation to heart disease $[1,30,31]$. Numerous studies 
have documented the implications of miRNAs in nearly every developmental and pathologic process of the cardiovascular system, including cardiac arrhythmia, hypertrophy, fibrosis, ischemia, heart failure, and atherosclerosis. In this review we summarize the key miRNAs that can solely modulate the cardiovascular pathological process and discuss the mechanisms by which they exert their function and act as novel therapeutic targets and/or diagnostic markers. We will examine their role in ischemic heart disease; as diagnostic markers and therapeutic targets; critical modulators of cell differentiation, migration, proliferation, and apoptosis; their own transcriptional and posttranscriptional regulation; their critical role in cardiac remodeling; the function they play in regulation of vascular smooth muscle differentiation and response to injury; and the importance of a systems biology approach to understanding their involvement in the regulation of gene expression at every level of cardiovascular study.

A major obstacle in establishing miRNA-based clinical therapies is in the efficient delivery of miRNA mimics and inhibitors to target organs. There is little doubt that miRNAs play a significant role in cardiovascular development and disease, regulating cardiomyocyte self-renewal and differentiation, and normal cardiac structure. Of the 30 or more miRNAs that appear to be key factors in apoptosis, their potential gene network goes far beyond that small number [32]. miRNAs have complex gene regulation mechanisms, in part determined by the presence or absence of the target genes in a given cell type. Balancing and maintaining the threshold levels of these miRNAs is critical as a cell confronts its destiny to live or die. It is essential to identify the cross-talks between miRNA and other noncoding small RNAs such as Piwiinteracting RNA and repeat-associated small interfering RNA in understanding their multifaceted roles. miRNAs are critical effectors of cell regulation; and we are just beginning to understand their role as gatekeepers of both survival and cell death.

\section{References}

1. Barringhaus, K. G., \& Zamore, P. D. (2009). MicroRNAsregulating a change of heart. Circulation, 119, 2217-2224.

2. Gibbs, R. A. (2003). The International HapMap project. Nature, 426, 789-796.

3. Roberts, R. (2007). New gains in understanding coronary artery disease, Interview with Dr. Robert Roberts. Affymetrix Microarray Bulletin, 3, 1-4.

4. Nakamura, Y., Leppert, M., OConnell, P., Wolff, R., Holm, T., Culver, M., et al. (1987). Variable number of tandem repeat (VNTR) markers for human gene mapping. Science, 235, 1616-1622.

5. Cooperative Human Linkage Center (CHLC). (1994). A comprehensive human linkage map with centimorgan density. Science, 265, 2049-2054.
6. Hamosh, A., Scott, A. F., Amberger, J., Bocchini, C., Valle, D., \& McKusick, V. A. (2002). Online Mendelian Inheritance in Man (OMIM), a knowledgebase of human genes and genetic disorders. Nucleic Acids Research, 30, 52-55.

7. LaFramboise, T. (2009). Single nucleotide polymorphism arrays: a decade of biological, computational and technological advances. Nucleic Acids Research, 37, 4181-4193.

8. McPherson, R., Pertsemlidis, A., Kavaslar, N., Stewart, A. F. R., Cohen, J. C., Roberts, R., et al. (2007). A common allele on chromosome 9 associated with coronary heart disease. Science, 316, 1488-1491.

9. Helgadottir, A., Thorleifsson, G., Manolescu, A., Gretarsdottir, S., Blondal, T., Jonasdottir, A., et al. (2007). A common variant on chromosome 9p21 affects the risk of myocardial infarction. Science, 316, 1491-1493.

10. Goldstein, D. B. (2009). Common genetic variation and human traits. New England Journal of Medicine, 360, 1699-1701.

11. Jarinova, O., Stewart, A. F. R., Roberts, R., Wells, G., Lau, P., Naing, T., et al. (2009). Functional analysis of the chromosome 9 p21.3 coronary artery disease risk locus. Arteriosclerosis, Thrombosis and Vascular Biology, 29, 1671-1677.

12. Moulton, V. (2005). Tracking down noncoding RNAs. Proceedings of the National Academy of Sciences of the United States of America, 102, 2269-2270.

13. Tuschl, T. (2003). Functional genomics: RNA sets the standard. Nature, 421, 220-221.

14. Eulalio, A., Huntzinger, E., \& Izaurralde, E. (2008). Getting to the root of miRNA-mediated gene silencing. Cell, 132, 9-14.

15. Volinia, S., Calin, G. A., Liu, C. G., Ambs, S., Cimmino, A., Petrocca, F., et al. (2006). A microRNA expression signature of human solid tumors defines cancer gene targets. Proceedings of the National Academy of Sciences of the United States of America, 103, 2257-2261.

16. Iorio, M. V., \& Croce, C. M. (2009). MicroRNAs in cancer: small molecules with a huge impact. Journal of Clinical Oncology, 27, $5848-5856$.

17. Soifer, H. S., Rossi, J. J., \& Saetrom, P. (2007). MicroRNAs in disease and potential therapeutic applications. Molecular Therapy, 15, 2070-2079.

18. Altuvia, Y., Landgraf, P., Lithwick, G., Elefant, N., Pfeffer, S., Aravin, A., et al. (2005). Clustering and conservation patterns of human microRNAs. Nucleic Acids Research, 33, 2697-2706.

19. Bartel, D. P. (2009). MicroRNAs: target recognition and regulatory functions. Cell, 136, 215-233.

20. Eulalio, A., Huntzinger, E., \& Izaurralde, E. (2008). GW182 interaction with Argonaute is essential for miRNA-mediated translational repression and mRNA decay. Nature Structural \& Molecular Biology, 15, 346-353.

21. Yekta, S., Shih, I. H., \& Bartel, D. P. (2004). MicroRNA-directed cleavage of HOXB8 mRNA. Science, 304, 594-596.

22. Wightman, B., Ha, I., \& Ruvkun, G. (1993). Posttranscriptional regulation of the heterochronic gene lin-14 by lin- 4 mediates temporal pattern formation in C. elegans. Cell, 75, 855-862.

23. Lee, R. C., Feinbaum, R. L., \& Ambros, V. (1993). The $C$. elegans heterochronic gene lin-4 encodes small RNAs with antisense complementarity to lin-14. Cell, 75, 843-854.

24. Reinhart, B. J., Slack, F. J., Basson, M., Pasquinelli, A. E., Bettinger, J. C., Rougvie, A. E., et al. (2000). The 21-nucleotide let-7 RNA regulates developmental timing in Caenorhabditis elegans. Nature, 403, 901-906.

25. Pasquinelli, A. E., Reinhart, B. J., Slack, F., Martindale, M. Q., Kuroda, M. I., Maller, B., et al. (2000). Conservation of the sequence and temporal expression of let-7 heterochronic regulatory RNA. Nature, 408, 86-89.

26. Griffiths-Jones, S., Saini, H. K., van Dongen, S., \& Enright, A. J. (2008). miRBase: tools for microRNA genomics. Nucleic Acids Research, 36(Database Issue), D154-158. 
27. Berezikov, E., Guryev, V., van de Belt, J., Wienholds, E., Plasterk, R. H., \& Cuppen, E. (2005). Phylogenetic shadowing and computational identification of human microRNA genes. Cell, 120, 21-24.

28. Sheng, Y., Engström, P. G., \& Lenhard, B. (2007). Mammalian microRNA prediction through a support vector machine model of sequence and structure. PLoS One, 2, e946.

29. Friedman, R. C., Farh, K. K., Burge, C. B., \& Bartel, D. P. (2009). Most mammalian mRNAs are conserved targets of microRNAs. Genome Research, 19, 92-105.
30. Catalucci, D., Gallo, P., \& Condorelli, G. (2009). MicroRNAs in cardiovascular biology and heart disease. Circulation Cardiovascular Genetics, 2, 402-408.

31. Pan, Z-w, Lu, Y-j, \& Yang, B-f. (2010). MicroRNAs: a novel class of potential therapeutic targets for cardiovascular diseases. Acta Pharmacologica Sinica, 31, 1-9.

32. Subramanian, S., \& Steer, C. J. (2010). MicroRNAs as gatekeepers of apoptosis. Journal of Cellular Physiology, 223, 289298. 